Z Gerontol Geriat 2022 · 55:197-203 https://doi.org/10.1007/s00391-022-02021-x Eingegangen: 7. Dezember 2021 Angenommen: 7. Januar 2022

Online publiziert: 29. Januar 2022

(C) The Author(s), under exclusive licence to Springer Medizin Verlag GmbH, ein Teil von Springer Nature 2022

\section{Interprofessionelle Teambildung - ein hochschulübergreifendes Qualifizierungsprogramm}

\author{
Cornelia Kricheldorff' · Bernhard Heimbach ${ }^{2} \cdot$ Ines Himmelsbach ${ }^{3} \cdot$ Hauke Schumann $^{3}$ \\ 'Katholische Hochschule Freiburg (em.), Freiburg, Deutschland \\ ${ }^{2}$ Zentrum für Gerontologie und Geriatrie, Universitätsklinikums Freiburg, Freiburg, Deutschland \\ ${ }^{3}$ Katholische Hochschule Freiburg, Freiburg, Deutschland
}

Zusammenfassung

\section{In diesem Beitrag}

\footnotetext{
- Ausgangslage und Hintergrund

- Qualifizierungsprogramm Programmtag 1 (Umfang 8 Unterrichtseinheiten) - Programmtag 2 (Umfang 8 Unterrichtseinheiten)

- Erfahrungen und Ergebnisse

- Entwicklungen und aktueller Stand

- Diskussion der Ergebnisse
}

\begin{abstract}
Hintergrund: Mit der wachsenden Zahl älterer und alter sowie von Multimorbidität, kognitiven Einschränkungen und Frailty betroffener Patient*innen im Krankenhaus und der Ausweitung der Langzeitpflege steigen die Herausforderungen in geriatrischen und gerontologischen Versorgungssettings. Sich verändernde Familienstrukturen lassen soziale Netzwerke und Ressourcen brüchig werden. Diese Entwicklung erfordert eine starke interprofessionelle Teambildung und Vernetzung der relevanten Akteure im Pflege- und Gesundheitswesen.

Ziele: Ein Qualifizierungsprogramm für Studierende der Medizin, der Sozialen Arbeit und pflegerelevanter Studiengänge wurde etabliert. Teilnehmende sollen hierin gemeinsam lernen, sich fallbezogen mit Anliegen und Bedarfen geriatrischer Patient*innen zu beschäftigen sowie geeignete Behandlungs- und Interventionspläne zu entwickeln.

Methode: Das Qualifizierungsprogramm zur interprofessionellen Teambildung wurde in der Förderphase als Pilotvorhaben erprobt und mithilfe des Freiburger Fragebogen Interprofessionelle Lernevaluation (FILE) wissenschaftlich evaluiert $(n=78)$.

Ergebnisse: Bei rund $98 \%$ der Befragten erfährt das Programm eine hohe Zustimmung. Die Veränderungsmessung zeigt verbesserte Teamkompetenz und -fähigkeit.

Schlussfolgerung: Studierenden unterschiedlicher Fachrichtungen und Professionen bereits im Studium ein Lernfeld für interprofessionelles Lernen und Arbeiten zu eröffnen, schafft eine gute Basis für eine gelingende interprofessionelle Teambildung in der Fach- und Berufspraxis. Das vorgestellte hochschulübergreifende und interprofessionelle Lehrprojekt ist inzwischen mit dem Lehrmodul „Der geriatrische Patient" an den beteiligten Hochschulen curricular fest verankert. Es ist damit ein mögliches Modell für ähnliche Vorhaben.
\end{abstract}

\section{Schlüsselwörter}

Interprofessionelle Lehre · Interprofessionelle Teambildung · Kompetenzbasierte Ausbildung · Geriatrie $\cdot$ Gerontologie

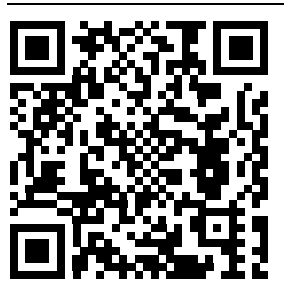

QR-Code scannen \&Beitrag online lesen

\section{Ausgangslage und Hintergrund}

Eine zentrale Herausforderung, mit der das deutsche Gesundheitswesen und das Versorgungssystem für Menschen mit Hilfeund Pflegebedarf schon heute und künftig noch viel stärker konfrontiert sein wird, ergibt sich aus dem deutlichen Anstieg der Zahl hochaltriger Menschen. Der Alters- aufbau in Deutschland wird sich künftig noch weiter in Richtung alter und hochaltriger Bevölkerungsgruppen verschieben. Dabei resultiert der Anstieg der Lebenserwartung maßgeblich aus dem Sinken der Mortalität v.a. in höheren und hohen Altersjahren [6]. Auch in der 14. koordinierten Bevölkerungsvorausberechnung für Deutschland werden Entwicklungen 
skizziert, die bis zum Jahr 2060 eine weitere moderate Erhöhung der durchschnittlichen Lebenserwartung prognostizieren. Lag im Jahr 2019 die fernere Lebenserwartung für 65-jährige Männer bei 17,9 weiteren Lebensjahren und bei gleichaltrigen Frauen bei 21,1 Jahren, können Männer im Jahr 2060 dann mit prognostizierten 21,8 weiteren Jahren rechnen, während Frauen dieses Alter noch 24,5 weitere Jahre zu erwarten haben [14].

Mit dem höheren Lebensalter zeigen sich häufiger und immer deutlicher die typischen Merkmale geriatrischer Patient*innen wie beispielsweise das Phänomen Frailty mit Auswirkungen auf Alltagskompetenz, Mobilität, seelisches Wohlbefinden und Kognition [11, 13], und es steigt auch das (statistische) Risiko von demenziellen Erkrankungen, Multimorbidität und Pflegebedürftigkeit [13]. Damit gehen häufig deutliche Einschränkungen bei Aktivitäten und sozialer Teilhabe einher, sowie die zunehmende Gefährdung alltagsrelevanter Selbstständigkeit [3].

Fachliche Antworten darauf müssen verstärkt auf die Schnittstellen im Versorgungssystem achten, diese bei der Interventionsplanung berücksichtigen [8] und auch die verschiedenen Facetten des Lebens im Alter miteinbeziehen. Und diese sind eben deutlich vielfältiger als die ausschließliche Fokussierung auf die medizinische Perspektive, in der Regel zumeist unterstützt durch gezielte physio- und ergotherapeutische Behandlung [13] und aktivierend-therapeutische Pflege [1]. Allerdings finden in dieser eng fokussierten Betrachtungsweise eben gerade die lebensweltlichen und sozialen Bezüge des alten Menschen zu wenig Berücksichtigung. Deshalb muss sich das "geriatrische Team", wie es sich in den letzten Jahrzehnten in Deutschland als ein von der Medizin dominiertes und koordiniertes Vorgehen im klinischen Alltag etabliert hat [17], zum wirklichen interprofessionellen Arbeiten wandeln, um befriedigende Antworten auf die sich verändernden und verdichtenden Bedarfe alter Menschen in der geriatrischen Praxis zu generieren. Angesichts der skizzierten Entwicklungen ist das „interprofessionelle Team" in der Geriatrie unbedingt auch durch die Perspektive von Sozialer Arbeit und Beratung zu erweitern, und das ent- sprechende Aufgabenspektrum muss sich hinsichtlich der berufsspezifischen Kompetenzen erweitern [7, 18]. Das bedeutet, dass klassische Rollenzuweisungen in der geriatrischen Versorgungsrealität künftig deutlicher hinterfragt werden und - damit einhergehend - Zuständigkeiten weniger starr festgeschrieben sein müssen. Dieser Aspekt wird durch aktuelle Studien weiter untermauert, da in geriatrischen Versorgungskontexten alte Menschen ihre zunehmende psychophysische Vulnerabilität mit Blick auf Einsamkeit, steigende Symptomlast, Gebrechlichkeit und den damit verbundenen Autonomieverlust wahrgenommen und von Professionellen adressiert wissen wollen [10].

Damit eine solchermaßen geprägte interprofessionelle Teambildung in der Praxis gelingt, braucht es einerseits organisationales Lernen in der geriatrischen Versorgung [15] und auch die entsprechend frühe Vorbereitung des Berufsnachwuchses in allen relevanten Fachrichtungen. In dieser Logik müssen bereits Studierende und Schüler*innen in definierten Modulen und speziellen Lehrangeboten auf die Wahrnehmung ihrer je eigenen Rolle im zunächst multiprofessionellen Team vorbereitet werden. Im Sinne einer Selbstvergewisserung und Klarheit in Bezug auf die eigenen Kompetenzen, Ressourcen und Stärken ist das dann die Basis für die Weiterentwicklung zum interprofessionellen Team, in dem die verschiedenen Akteure und Berufsgruppen auch über- und voneinander lernen können. Dieses deutlich erweiterte Verständnis von Teamarbeit auf Augenhöhe, getragen von den Kompetenzen, Stärken und Ressourcen aller beteiligten Professionen, ist bislang im deutschen Gesundheitssystem allenfalls in ersten Ansätzen verankert. Ein Beispiel, in dem solche Strukturen in der Praxis schon länger verankert sind, ist der Palliativbereich [2].

Und auch im Bereich der medizinischen Lehre gibt es modellhafte Beispiele, die bei den Studierenden auf sehr positive Resonanz stoßen, wie eines der wenigen Beispiele aus München zeigt, das ganz aktuell im GMS Journal for Medical Education veröffentlicht wurde [16]. Den Evaluationsergebnissen dieses Lehrprogramms folgend, betonen drei Viertel der befragten Teilnehmer*innen die für sie hohe Bedeutung der interprofessionellen Lehre, und sie wünschen sich regelhaft mehr davon, um besser auf die Berufspraxis vorbereitet zu werden. Diese Forderung ist nicht neu, bestimmt aber bisher v.a. im internationalen Kontext die Fachdebatten [5, 12], vorrangig im Kontext von Community-basierten Ansätzen der Gesundheitsversorgung [21].

Eine Expertengruppe der WHO hat bereits in der zweiten Hälfte der 1980erJahre die große Bedeutung der interprofessionellen Lehre betont und unter der Orientierung "Learning together to work together for health" in einem Report dargelegt [20].Zur Bewertung der Qualität der Arbeit im interprofessionellen Team wurde die McMaster-Ottawa Scale entwickelt, die mit einer 3-stufigen Bewertungsskala zentrale und für das interprofessionelle Team konstitutive Elemente misst, was im Ergebnis zu einem individuellen Rating führt ([11], - Tab. 1).

In dieser Logik wurde in Kanada bereits 2010 ein nationaler Kompetenzrahmen für die Arbeit in interprofessionellen Teams im Gesundheitsbereich entwickelt und eingeführt [4]. Die jeweils als zentral benannten Kompetenzen für eine erfolgreiche Implementierung von interprofessionellen Teams sind in allen internationalen Tools und Quellen vergleichbar - es geht um Kommunikation und Kooperation auf Augenhöhe, basierend auf Rollenklarheit und Respekt zwischen den im Team beteiligten Akteuren und Berufsgruppen.

Genau daran anknüpfend, sind das Lernen voneinander und das kritische Reflektieren miteinander auch die Kernanliegen des hier vorgestellten Qualifizierungsprogramms in Freiburg, in dem, über Hochschul- und Fachgrenzen hinweg, Studierende der Medizin, der Sozialen Arbeit und von pflegerelevanten Studiengängen, wie Pflegewissenschaften und Berufspädagogik im Gesundheitswesen, gemeinsam lernen, sich fallbezogen mit den Anliegen und Bedarfen geriatrischer Patient*innen zu beschäftigen und geeignete Behandlungs- und Interventionspläne zu entwickeln.

\section{Qualifizierungsprogramm}

Das im Beitrag vorgestellte Lehrprojekt wurde vom Ministerium für Wissenschaft, Forschung und Kunst Baden-Württemberg 
Tab. 1 Bewertung interprofessioneller Kompetenzen nach der McMaster-Ottawa Scale. (Eigene Darstellung in Anlehnung an Lie et al. [12])

\begin{tabular}{|c|c|c|c|}
\hline Competencies & $\begin{array}{l}\text { Below } \\
\text { expected }\end{array}$ & $\begin{array}{l}\text { At expec- } \\
\text { ted }\end{array}$ & $\begin{array}{l}\text { Above } \\
\text { expected }\end{array}$ \\
\hline Communication & \multirow[t]{4}{*}{1} & \multirow[t]{4}{*}{2} & \multirow[t]{4}{*}{3} \\
\hline Assertive communication & & & \\
\hline Respectful communication & & & \\
\hline Effective communication & & & \\
\hline Collaboration & \multirow[t]{4}{*}{1} & \multirow[t]{4}{*}{2} & \multirow[t]{4}{*}{3} \\
\hline Establishes collaborative relationships & & & \\
\hline Integrates different perspectives & & & \\
\hline Ensures shared information & & & \\
\hline Roles and responsibilities & \multirow[t]{3}{*}{1} & \multirow[t]{3}{*}{2} & \multirow[t]{3}{*}{3} \\
\hline Describes roles and responsibilities & & & \\
\hline Shares knowledge with others; accepts accountability & & & \\
\hline Collaborative patient-family centered approach & \multirow[t]{3}{*}{1} & \multirow[t]{3}{*}{2} & \multirow[t]{3}{*}{3} \\
\hline Seeks input from patient and family & & & \\
\hline Shares with patient and family & & & \\
\hline Conflict management resolution & \multirow[t]{4}{*}{1} & \multirow[t]{4}{*}{2} & \multirow[t]{4}{*}{3} \\
\hline Demonstrates active listening & & & \\
\hline Respects different perspectives & & & \\
\hline Works with others to prevent conflicts & & & \\
\hline Team functioning & \multirow[t]{4}{*}{1} & \multirow[t]{4}{*}{2} & \multirow[t]{4}{*}{3} \\
\hline Evaluates team function and dynamics & & & \\
\hline Contributes effectively & & & \\
\hline Demonstrates shared leadership & & & \\
\hline Global rating score & 1 & 2 & 3 \\
\hline
\end{tabular}

(MWK Baden-Württemberg) zunächst von 2016 bis 2018 als Modell gefördert, in kleinen Gruppen als Pilotvorhaben erprobt und in dieser Zeit wissenschaftlich begleitet und auch mit einem speziellen Instrument evaluiert [9]. Zur Verstetigung des Programms gab es für 2 weitere Jahre eine entsprechende Landesförderung, die inzwischen zu einer curricularen Verankerung des Lehrprogramms „Der geriatrische Patient" in geeigneten Modulen an den beiden beteiligten Hochschulen geführt hat. Es richtet sich als inhaltliche Schwerpunktsetzung und Vertiefung an Studierende der Medizin an der Universität Freiburg und der Angewandten Pflegewissenschaft, der Berufspädagogik im Gesundheitswesen und der Sozialen Arbeit an der Katholischen Hochschule Freiburg. In der gemeinsamen Lehre geht es v.a. um die Vermittlung von Kenntnissen und ihre fallbezogene Anwendung, teilweise in Verbindung mit dem Einsatz von Simulationspatient*innen, in Teams von Studierenden, die in einer multiprofessionellen Zusammensetzung gebildet werden.
Die Struktur des Programms, das insgesamt 16 Unterrichtseinheiten (UE) umfasst, ist in 4 Teile gegliedert. Diese werden an 2 ganzen Tagen (je 8UE), die im Abstand von 2 bis 3 Wochen stattfinden, angeboten. In der Zeit zwischen den beiden Tagen im Seminar arbeiten und verständigen sich die Studierenden fallbezogen in den multiprofessionell zusammengestellten Teams. Die 4 Teile umfassen folgende Inhalte:

\section{Programmtag 1 (Umfang 8 Unterrichtseinheiten)}

Teil A: gemeinsame Einführungsvorlesung - Grundlagen der Gerontologie und Geriatrie.

Teil B: 4 Workshops, die im Rotationsprinzip besucht werden, also durchlaufen alle Studierenden gestaffelt alle 4 Angebote. Dabei geht es um das Kennenlernen ...

- einer aktiven „interprofessionellen Früherkennungsstrategie" zur Identifikation des geriatrischen Patienten und seiner alltagsrelevanten Einschrän- kungen auf der Basis verschiedener Instrumente, wie Geriatrie-Check und Geriatrisches Assessment [8],

- von Selbsterfahrung zur Verbesserung von Empathie und einer positiveren Einstellung gegenüber dem Alter durch den Einsatz von „Alterssimulationsanzügen" (Perspektivenwechsel), um den Studierenden verschiedene altersbedingte Phänomene und Einschränkungen erfahrbar zu machen [19],

- möglicher professioneller Fallstricke und Hürden, unter Berücksichtigung der häufigsten geriatrischen Syndrome, auf der Basis von Fallbeispielen [13],

- relevanter sozialrechtlicher Aspekte, z. B. Kompetenzen zur Beratung bei Pflegebedarf sowie bei der Beantragung eines Pflegegrads, einer Rehamaßnahme oder eines Behindertenausweises [18].

\section{Programmtag 2 (Umfang 8 Unterrichtseinheiten)}

Teil C: fallbezogenes Arbeiten in interprofessionellen Arbeitsgemeinschaften (AG) und gemeinsame Erarbeitung einer Präsentation.

Teil D: gemeinsame Auswertung der Erfahrungen im Plenum.

\section{Erfahrungen und Ergebnisse}

Die Lernerfahrungen in den Kursen der ersten Förderphase durch das MWK Baden-Württemberg wurden mit dem spezifischen Evaluationsinstrument Freiburger Fragebogen Interprofessionelle Lernevaluation (FILE, [9]) in einer Prä-post-Befragung wissenschaftlich erfasst und statistisch ausgewertet. In allen folgenden Kursen kamen die Standardinstrumente zur Lehrevaluation an der Universität Freiburg und an der Katholischen Hochschule Freiburg zum Einsatz. Das bedeutet, dass sich die statistischen Auswertungen der bei den Studierenden erhobenen Daten, auf die hier in den Tabellen und Abbildungen Bezug genommen wird, v. a. auf die Kurse der ersten Förderphase beziehen, die vom Wintersemester 2016/2017 bis einschließlich Sommersemester 2018 liefen. Ergänzt werden diese Erkenntnisse aber zusätzlich durch die Auswertungen der Lehrevalua- 
Tab. 2 Verteilung der Studierenden nach Geschlecht

\begin{tabular}{|l|l|l|l|l|l|}
\hline- & Anzahl $(n)$ & Anteil (\%) & Gültige Prozente & Kumulierte Prozente \\
\hline \multirow{2}{*}{ Gültig } & Weiblich & 39 & 75,0 & 76,5 & 76,5 \\
\cline { 2 - 6 } & Männlich & 12 & 23,1 & 23,5 & 100,0 \\
\cline { 2 - 6 } & Gesamt & 51 & 98,1 & 100,0 & - \\
\hline Fehlend & -10 & 1 & 1,9 & - & - \\
\hline Gesamt & - & 52 & 100,0 & - & - \\
\hline
\end{tabular}

\begin{tabular}{|l|l|l|}
\hline \multicolumn{2}{|l|}{ Tab. 4 Studiensemester bei Teilnahme } \\
\hline$n$ & Gültig & $\mathbf{5 1}$ \\
\cline { 2 - 3 } & Fehlend & $\mathbf{1}$ \\
\hline Mittelwert & 5,75 \\
\hline Standardabweichung & $\pm 3,045$ \\
\hline Minimum & 2 \\
\hline Maximum & 12 \\
\hline
\end{tabular}

tion, die seitdem regelmäßig in jedem Semester stattfinden.

An den 4 Kursen der ersten Förderphase haben insgesamt 78 Studierende teilgenommen. Vollständige und damit auswertbare Ergebnisse im Prä-post-Vergleich liegen von 52 Teilnehmenden vor. Aufgrund einzelner fehlender Abgaben variiert aber die Anzahl der einbezogenen Evaluationsbogen um +1 oder -1 . Die Auswertung der Verteilung der Studierenden nach Geschlecht, Alter und Anzahl der bereits absolvierten Studiensemester im jeweiligen Fach ergibt folgendes Bild:

In der deskriptiven Statistik (- Tab. 2, 3 und 4) zeigt sich, dass die Studierenden, die an den Pilotdurchgängen des Qualifizierungsprogramms zur interprofessionellen Teambildung in der Geriatrie teilgenommen haben, zu 75\% weiblich waren. Im Mittel waren sie ca. 30 Jahre alt, bereits in ihrem 6. Studiensemester und brachten damit auch schon z.T. sehr fundierte Praxiserfahrungen mit.

Sichtbar wird auch ein sehr breiter Altersrange, was v.a. an den oft schon älteren und parallel zur eigenen Berufstätigkeit Studierenden in den pflegerelevanten Studiengängen liegt. Und bemerkbar macht sich auch die Tatsache, dass alle Studierenden der Humanmedizin schon kurz vor Abschluss ihres Studiums (überwiegend im 9. Semester) standen. Die Verteilung nach Studiengängen war wie folgt:

DieTeilnehmer*innen aus dem Studiengang Humanmedizin an der Universität
Freiburg (37\%) und der Sozialen Arbeit an der Katholischen Hochschule Freiburg (35\%) waren in der Pilotphase der Qualifizierung die beiden großen Gruppen und damit fast gleich stark. Aus den pflegerelevanten Studiengängen kam ein Viertel der Studierenden (• Abb. 1).

Damit trafen in den interprofessionellen Teams, die sich gemeinsam mit der Fallarbeit beschäftigten, Berufsgruppen aufeinander, die beispielsweise in der klinischen Praxis oft weniger fachliche Schnittstellen haben. Und genau darin lag für die teilnehmenden Studierenden auch der subjektive Wert, der insgesamt sehr hoch eingeschätzt wurde, wie sich in $\bullet$ Abb. 2 deutlich zeigt.

\section{Entwicklungen und aktueller Stand}

Das Lehrmodul hat sich mittlerweile an der Medizinischen Fakultät der Universität Freiburg und an der Katholischen Hochschule Freiburg etabliert und ist curricular fest verankert. Seit Auslaufen der Pilotphase wird es regelhaft in jedem Semester angeboten und in den Räumen der Katholischen Hochschule durchgeführt. Die Beteiligung liegt pro Durchgang bei 25 bis 35 Studierenden aus allen genannten Studienfächern, die in den 4 Workshops und zur Fallbearbeitung in Teams so aufgeteilt werden, dass alle professionellen Perspektiven entsprechend vertreten sind.

In den Semestern, die durch die "Coronavirus-disease-2019"(COVID-19)-Pandemie geprägt waren und in denen nur Online-Lehre möglich war, wurde das Modul in reduzierter und angepasster Form durchgeführt, wobei die praktischen Teile, v.a. die Selbsterfahrung mit dem Alterssimulationsanzug nicht wie skizziert durchgeführt werden konnten. Es war dem Team der Lehrenden aber wichtig, auch in Pandemiezeiten Kontinuität zu
Tab. 3 Altersrange und Mittelwert der Teilnehmenden

\begin{tabular}{|l|l|l|}
\hline$n$ & Gültig & $\mathbf{5 1}$ \\
\cline { 2 - 3 } & Fehlend & $\mathbf{1}$ \\
\hline Mittelwert & 30,65 \\
\hline Standardabweichung & $\pm 10,465$ \\
\hline Minimum & 19 \\
\hline Maximum & 58 \\
\hline
\end{tabular}

zeigen und damit deutlich zum Ausdruck zu bringen, dass es sich bei dem entwickelten Lehrmodul um einen relevanten und konstitutiven Ansatz und Ausdruck zukunftsweisender Versorgungsrealität im Gesundheitsbereich handelt - also nicht um verzichtbare Kür, sondern Pflicht!

Wichtig ist auch, dass die Zusammensetzung der beteiligten Lehrenden dem Muster des interprofessionellen Teams entspricht. Das bedeutet, dass aufseiten der Professor*innen die Perspektiven von Sozialer Arbeit, Sozialer Gerontologie, Geriatrie, Pflege und medizinischer Lehrdidaktik vertreten sind. In die 4 Workshops, die alle Studierendenteams durchlaufen, bringen Mitarbeitende aus dem Zentrum für Gerontologie und Geriatrie (ZGGF) des Universitätsklinikums Freiburg zusätzlich den Blick aus der einschlägigen Praxis ein. Das bedeutet, dass hier Lehrtandems zusammenarbeiten, in denen auch die Sozialarbeiterin, der Physiotherapeut und die Pflegefachkraft jeweils themenbezogen und eng abgestimmt mit den Professor*innen kooperieren und so einen direkten Anwendungsbezug garantieren. In der Planung und Durchführung bedeutet das umfangreiche Koordinationsarbeit, und es ist ein großer personeller Aufwand für eine relativ kleine Gruppe von Studierenden, der sich aber lohnt. Die Ergebnisse der Lehrevaluation sprechen für sich und decken sich noch immer mit den Bewertungen in der Evaluation der Pilotphase. Das trifft ganz besonders auf die eigene Rollenklärung im interprofessionellen Team zu, und ganz entscheidend ist auch, dass die fachlich breit aufgestellte Gruppe der Lehrenden als Modell für interprofessionelle Teambildung steht.

\section{Diskussion der Ergebnisse}

Das hochschulübergreifende Freiburger Qualifizierungsprogramm überschreitet 


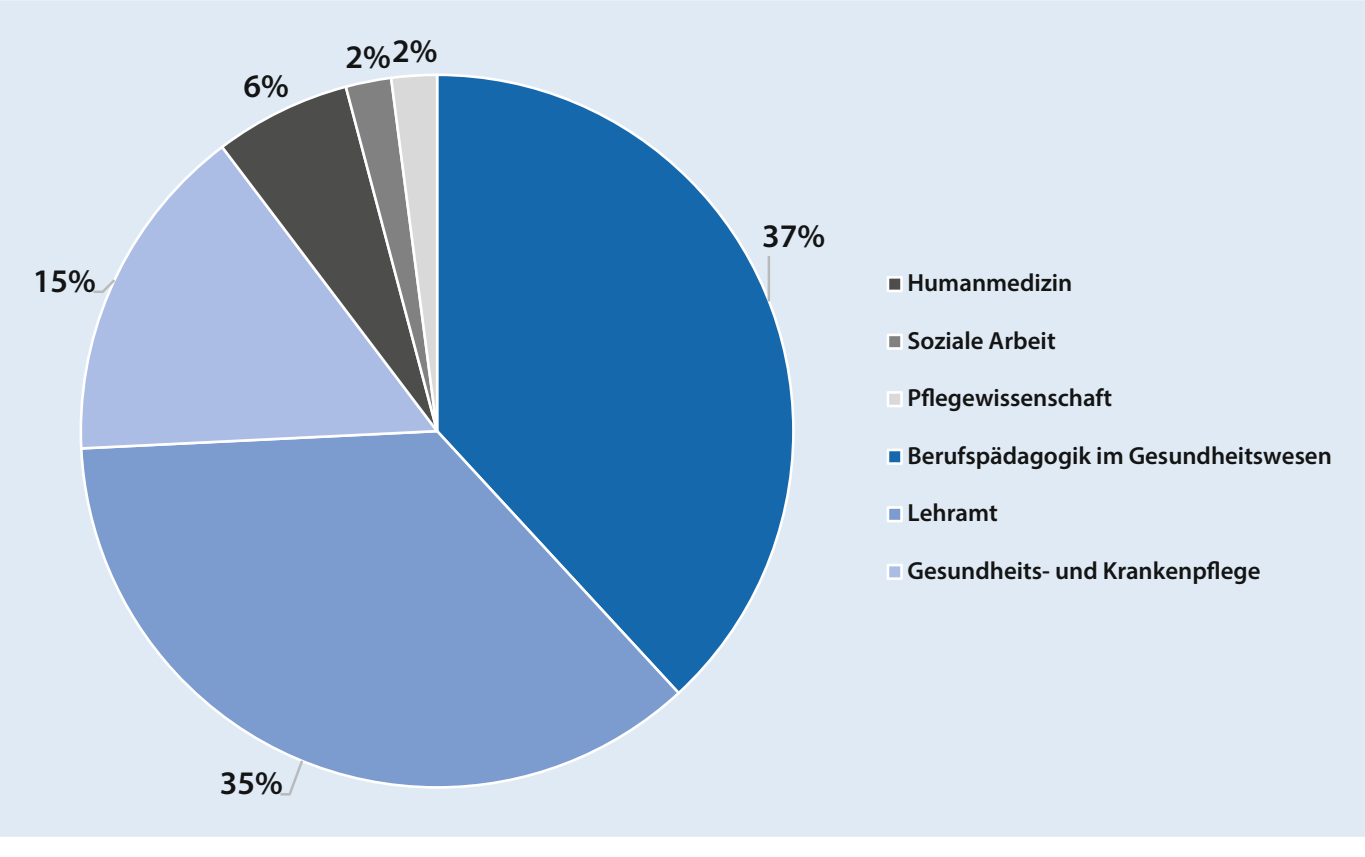

Abb. $1 \varangle$ Verteilung der Teilnehmenden nach Studiengängen

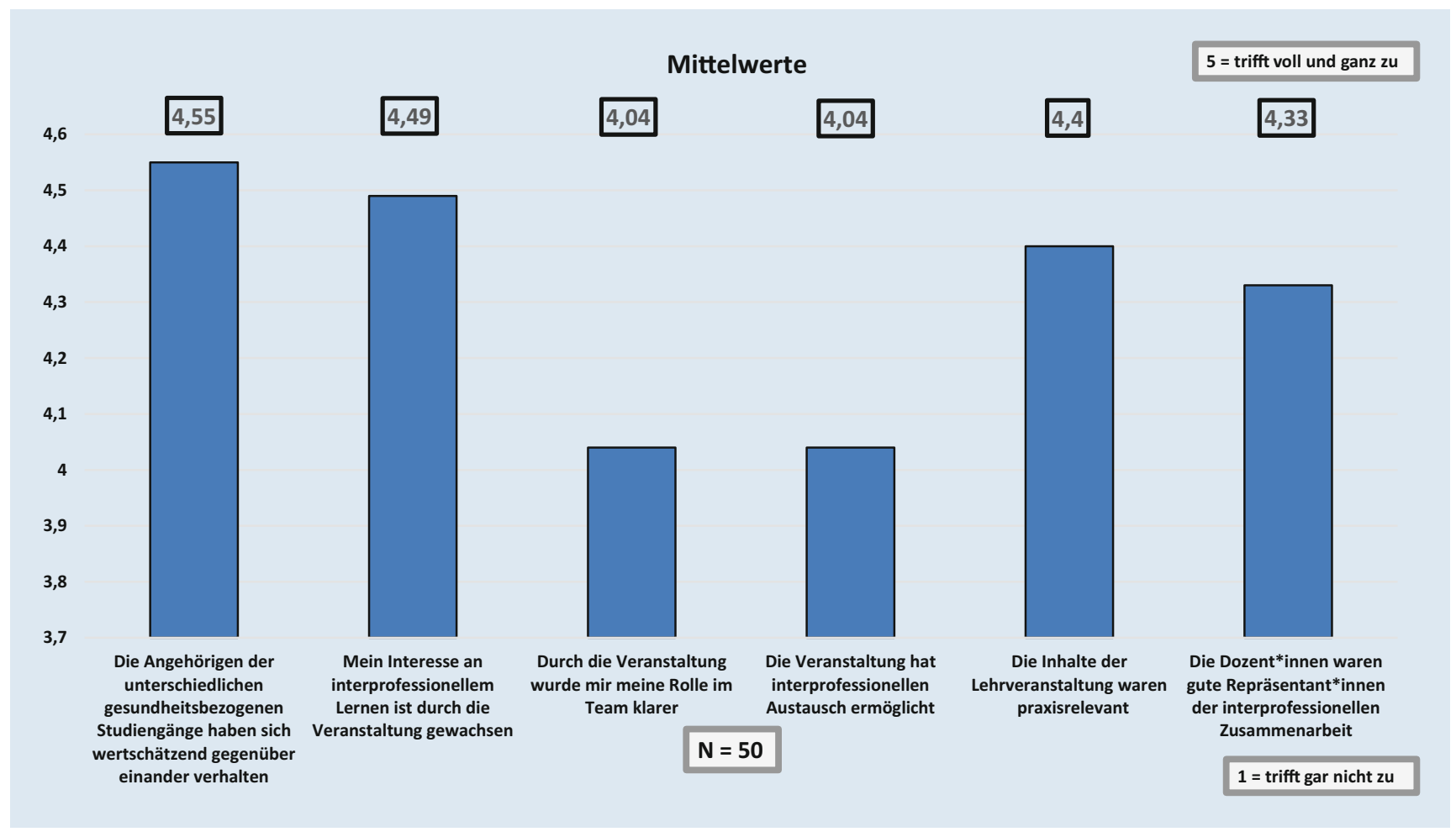

Abb. $2 \Delta$ Einschätzungen der Teilnehmenden zu den Wirkungen ihrer Teilnahme am Qualifizierungsprogramm

in Aufbau und Ausrichtung die meisten der curricularen Bausteine im Medizinstudium deutlich, die häufig nur auf ein Team von Vertreter*innen verschiedener fachärztlicher Ausrichtungen oder auf die multidisziplinäre Zusammenarbeit mit therapeutischen Fachkräften und/oder der Pflege beschränkt sind.
Diese Weitung des Teamverständnisses auch auf Berufsgruppen, die im geriatrischen Setting zusätzlich die für alte Menschen besonders wichtige soziale Perspektive einbringen, ist neu. Und das ist auch das innovative Moment im hier vorgestellten Modul „Der geriatrische Patient", weil damit auch ein sektorenübergreifen- des Denken und Handeln angeregt wird, das in der Realität der Praxis dringend gebraucht wird, wie dies auch in jüngsten Arbeiten bestätigt werden konnte [10].

So zeigen beispielsweise auch die aktuell veröffentlichten Evaluationsergebnisse zu den Wahlcurricula im Medizinstudium an der Ludwig-Maximilians-Univer- 
sität (LMU) München, dass Studierende immer deutlicher den Anspruch formulieren, „über ihren Tellerrand“ hinaussehen zu wollen und sich dafür Angebote wünschen, die ihr kreatives $(53,6 \%)$, kritisches $(63,7 \%)$ und interdisziplinäres Denken $(69 \%)$ anregen [15], um damit insgesamt bessere Ärzte zu werden (87\%). Dieses Votum ist eindeutig, muss aber über die Fachgrenzen der Medizin hinaus erweitert und umgesetzt werden, denn das verbindet zunächst eher gegenseitig fremde Denklogiken miteinander, und es entstehen mehr Offenheit, Wertschätzung und eine bessere Kommunikation. Und das wiederum schafft die Basis für eine interprofessionelle Teambildung, die nicht nur in der geriatrischen Praxis dringend gebraucht wird.

\section{Fazit für die Praxis}

Interdisziplinäre Teambildung ...

- verbessert die Versorgungsrealität und -praxis nicht nur im Bereich der Geriatrie, hat hier aber in Bezug auf die komplexe Lebensrealität alter Menschen eine besondere Relevanz.

- muss bereits im Studium oder in der Ausbildung anregt und entwickelt werden, um das enge disziplinäre Denken schon beim Berufsnachwuchs zu überwinden.

- braucht deshalb eine deutliche curriculare Verankerung in den Studien- und Ausbildungsprogrammen.

- ist auch eine organisationale Aufgabe und braucht deshalb ermöglichende Strukturen und Voraussetzungen.

\section{Korrespondenzadresse}

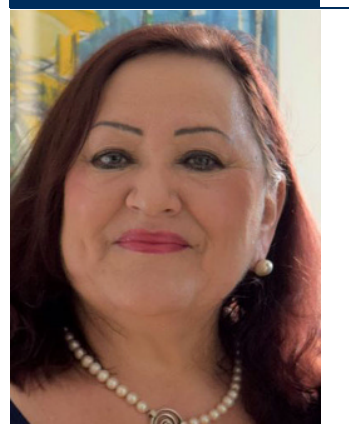

Prof. Dr. Cornelia Kricheldorff

Katholische Hochschule Freiburg (em.) Barbarastr. 7, 79106 Freiburg, Deutschland cornelia.kricheldorff@t-online.de

Förderung. Das im Beitrag vorgestellte hochschulübergreifende und interprofessionelle Lehr-

\section{Interprofessional team building-An interuniversity qualification program}

Background: With the growing number of older and old patients as well as patients affected by multimorbidity, cognitive impairments and frailty in hospital and expansion of long-term care, the challenges in the various geriatric and gerontological care settings are also increasing. Social networks and resources become fragile due to the changing family structures. A strong interprofessional team building and networking of the main actors in the nursing and healthcare systems become necessary.

Objective: A qualification program for students of medicine, social work and relevant study courses for nursing was established. In this program participants should collectively learn to deal with the concerns and needs of geriatric patients in a caserelated manner and to develop suitable plans for treatment and interventions.

Method: The qualification program for interprofessional team building was evaluated during the development phase as a pilot project and scientifically evaluated $(n=78)$ using the Freiburg questionnaire on interprofessional learning evaluation (FILE).

Results: The program experienced a high level of approval by approximately $98 \%$ of the participants. The measurement of change showed an improvement in team skills and ability to work in a team.

Conclusion: Opening up a learning field for interprofessional learning and working to students of different disciplines and professions during their studies creates a good basis for successful interprofessional team building in the subsequent professional practice. The interuniversity and interprofessional teaching project presented is now firmly anchored in the curriculum at the participating universities with the teaching module "The geriatric patient". It is therefore a possible model for similar projects.

\section{Keywords}

Interprofessional education - Interprofessional team building - Competency-based education . Geriatrics · Gerontology

projekt wurde vom Ministerium für Wissenschaft, Forschung und Kunst Baden-Württemberg in den Jahren 2016-2020 gefördert.

\section{Einhaltung ethischer Richtlinien}

Interessenkonflikt. C. Kricheldorff, B. Heimbach, I. Himmelsbach und H. Schumann geben an, dass kein Interessenkonflikt besteht.

Für diesen Beitrag wurden von den Autoren keine Studien an Menschen oder Tieren durchgeführt. Für die aufgeführten Studien gelten die jeweils dort angegebenen ethischen Richtlinien.

\section{Literatur}

\section{Verwendete Literatur}

1. Bartels F (Hrsg) (2021) Pflege und Therapie im interdisziplinären Team, 1.Aufl.Bd.5.Kohlhammer, Stuttgart

2. Bausewein C, Roller S (2018) Multiprofessionelles und interdisziplinäres Team. In:Leitfaden Palliative Care. Elsevier, München

3. BeyM (2011)Geriatrische Rehabilitation.Leitfaden für die Pflegepraxis, 1. Aufl. Kohlhammer, Stuttgart

4. CIHC-Canadian Interprofessional Health Collaborative (2010) A national interprofessional competency framework, University of British Columbia.
http://www.cihc.ca/files/CIHC_IPCompetencies Feb1210.pdf.Zugegriffen: 15. Nov. 2021

5. Croker A, Brown L, Little A, Crowley E (2016) Interprofessional relationships for work-integrated learning in healthcare: identifying scope for ongoing professional development. Creative Educ 7:1729-1738. https://doi.org/10.4236/ce.2016. 712176

6. Destatis - Statistisches Bundesamt (2021) Pressemitteilung Nr. N 041 vom 24. Juni 2021. https:// www.destatis.de/DE/Presse/Pressemitteilungen/ 2021/06/PD21_N041_12.html. Zugegriffen: 15. Nov. 2021

7. Duca Palmer S (2021) Social work and geriatric services. Palm Bay (USA). Apple Academic Press, Burlingtin

8. Freund H (2017) Geriatrisches Assessment und Testverfahren. Grundbegriffe - Anleitungen - Behandlungspfade, 3. Aufl. Kohlhammer, Stuttgart (http://www.content-select.com/index. php?id=bib_view\&ean=9783170326309. zuletzt abgerufen am 15.11.2021)

9. Giesler M, Bergmann S (2016) Evaluation of interprofessional education with FILE. Freiburg Poster session presented at: AMEE Conference, Barcelona, 2016 Aug 28-31

10. Kanat M, Schaefer J, Kivelitz L, Dirmaier J, VoigtRadloffS, Heimbach B, Glattacker M (2021) Patientcenteredness in the multimorbid elderly: a focus group study. BMC Geriatr. https://doi.org/10.1186/ s12877-021-02448-8

11. Kuphal A (2019) Interdisziplinarität, therapeutisches Team und der Umgang mit den "Schnittstellen" sowie Erläuterungen zur "Aktivierend- 
therapeutischen Pflege“. In: Rehm M, Schwibbe W (Hrsg) Praxiswissen Geriatrie. Ältere Menschen multiprofessionell begleiten, 1. Aufl. Kohlhammer, Stuttgart, S91-99

12. Lie D, May W, Richter-Lagha R, Forest C, Banzali Y, Lohenry K (2015) Adapting the McMaster-Ottawa scale and developing behavioral anchors for assessing performance in an interprofessional Team Observed Structured Clinical Encounter. Med Educ Online. https://doi.org/10.3402/meo.v20. 26691

13. Musolf M (2019) Das multiprofessionelle geriatrische Team. In: Rehm M, Schwibbe W (Hrsg) Praxiswissen Geriatrie. Ältere Menschen multiprofessionell begleiten, 1. Aufl. Kohlhammer, Stuttgart, S72-79

14. RKI - Robert Koch Institut (2015) Gesundheit in Deutschland. Gesundheitsberichterstattung des Bundes. Gemeinsam getragen von RKI und Destatis. Berlin: RKI. https://www.destatis.de/ DE/Themen/Gesellschaft-Umwelt/Gesundheit/ Gesundheitszustand-Relevantes-Verhalten/ Publikationen/Downloads-Gesundheitszustand/ gesundheit-in-deutschland-publikation.pdf? blob=publicationFile.Zugegriffen: 15. Nov. 2021

15. Rölker-Denker L (2018) Organisationales Lernen in der geriatrischen Versorgung. Dissertation. http://oops.uni-oldenburg.de/3612/1/roeorg18. pdf.Zugegriffen: 15. Nov. 2021

16. Rohr SO, Gerhard A, Schmidt F, Eder J, Salvermoser L, Dimitriadis K, Fischer MR (2021) Über den Tellerrand hinaus: Studierende positiv gegenüber visionären Wahlcurricula im Medizinstudium eingestellt. GMS J Med Educ. https://doi.org/10.3205/ zma001515

17. Vogel W (2017) Das Geriatrische Team. Wie interprofessionelles Arbeiten gelingt. Hg. v. Johannes Pantel und Rupert Püllen. Kohlhammer, Stuttgart (Altersmedizin in der Praxis)

18. Vogel W (2020) Soziale Arbeit in der Geriatrie. In: Aner K, Karl U (Hrsg) Handbuch Soziale Arbeit und Alter. Springer VS, Wiesbaden, S179-186

19. Watkins, C; Higham, E; Townley, C; Gilfoyle, M (2018): Can the Adam, Rouilly AK060 age suit successfully simulate students' experience of balance problems and risk of falling in frail older adults? A pre- and post-intervention study. Poster 41, British Society of Gerontology 47th Annual Conference-Ageing in an Unequal World: Shaping Environments for the 21st Century. Online unter: https://virtual.oxfordabstracts. com/\#/event/public/28/submission/273, zuletzt abgerufen am 15. Nov. 2021.

20. WHO - World Health Organisation (1988) Learning together to work together for health. Report of a WHO Study Group on Multiprofessional Education of Health Personnel:theTeam Approach. World Health Organ Tech Rep Ser 769:1-72

21. WHO - World Health Organisation (1987) Community-based education of health personnel. Report of a WHO study group. World Health Organ Tech RepSer 746:1-89

\section{Weiterführende Literatur}

22. Ernst F, Lübke N, Meinck M, Renz JC (2020) Kompendium Begutachtungswissen Geriatrie, 4. Aufl.Springer, Berlin, Heidelberg 